of this evaluation was to identify whether CAARMS scores differ between patients diagnosed with autism and matched controls in York EIP.

Method. From their mental health records, we identified all patients in the service with a diagnosis of autism. We then compared the CAARMS scores, at the time of referral, to those of age matched controls (matched by being in the age range 16-30) without an autism diagnosis, using continuous sampling by date of referral.

Result. 14 patients in the service had a diagnosis of autism and had completed a CAARMS. CAARMS domains are all scored between 0 and 6 (indicating increasing severity or frequency). Compared to the age matched controls, autistic patients had a higher mean difference in their scores for 'Non-Bizarre Ideas' (mean difference of 0.86 for severity and 0.57 for frequency) and 'Disorganised Speech' (mean difference of 0.28 for severity and 0.57 for frequency). These results did not reach statistical significance which was unsurprising given the sample size. The gender split between groups was similar.

Conclusion. Our evaluation suggests a difference in CAARMS scores between patients in our service with a diagnosis of autism and those without. A larger study would be needed to confirm a statistically significant difference and multicentre results would be needed as evidence of generalisability. However, if such a difference were confirmed it might question the validity of CAARMS in autistic patients or suggest that modifications, perhaps in the form of reasonable adjustments to the questions or scoring, were needed to increase the validity in this population. We would suggest that spending extra time checking the patient has understood the intended meaning of the questions in the CAARMS may increase validity, particularly in the 'Non-Bizarre Ideas' domain.

\section{COVID-19 pandemic moral injury in healthcare professionals: a systematic review}

Verity Williams ${ }^{1 \star}$, Rhian Bradley ${ }^{1}$, Rafey Faruqui ${ }^{1}$, Julia Hynes ${ }^{2}$ and Julie Anderson ${ }^{3}$

${ }^{1}$ Kent and Medway NHS and Social Care Partnership Trust, Kent and Medway Medical School; ${ }^{2}$ Kent and Medway Medical School and

${ }^{3}$ University of Kent

${ }^{*}$ Corresponding author.

doi: 10.1192/bjo.2021.204

Aims. Moral injury (MI) refers to psychological distress resulting from witnessing or participating in events which violate an individual's moral code. Originating from military experiences, the phenomenon also has relevance for healthcare professionals dealing with wars, natural disasters and infectious diseases. The deontological basis of medicine prioritises duty to the individual patient over duty to wider society. These values may place healthcare professionals at increased risk of moral injury, particularly in crisis contexts where they may be party to decisions to withdraw or divert care based on resource availability.

We conducted a systematic review of medical literature to understand the extent and clinical and socio-demographic correlates of moral injury during the COVID-19 pandemic.

Method. We conducted a systematic review of reports included in MEDLINE, PsycINFO, BNI, CINAHL, EMBASE, EMCARE and HMIC databases using search terms: "moral injury" AND "covid" OR "coronavirus" OR "pandemic". We also searched Google Scholar and Ovid Database and conducted reference searching. We searched for published quantitative primary research as well as advance online publications and pre-print research. Findings are reported in line with Preferred Reporting Items for Systematic Reviews and MetaAnalyses (PRISMA). Two authors independently assessed the included studies' methodological quality using a seven-item checklist.

Result. Our databases search identified 498 records and other sources identified 4 records. We screened 391 records after removing duplicates. 4 reports met our protocol requirements.

Three papers used cross-sectional designs. One reported longitudinal outcomes of their sample already described in one of the three papers. Only one study used a MI scoring system validated for healthcare professionals. Others used scoring validated in military populations. These papers reported outcomes from 3334 subjects, with a higher proportion of females. The largest study (3006 subjects) reported MI in $41.3 \%$ of their sample. Overall, factors associated with greater MI included: providing direct care to COVID-19 patients; sleep troubles; being unmarried; aged <30 years; female gender; and Buddhist/Taoist faith. Nurses reported a greater severity of MI than physicians. MI significantly correlated with anxiety, depression and burnout. The longitudinal study reported that more stressful and less supportive work environments predicted greater MI at 3 months follow-up.

The average quality assessment score of these studies was 4/7. Conclusion. It is important that we are able to address moral injury awareness training as part of workforce preparedness and burnout prevention during the COVID-19 pandemic and other disaster responses across the globe.

\section{Do Junior Doctors feel confident using Emergency Detention Certificates?}

Sarah Wordie ${ }^{*}$ Alice Troup, Giovana Klefti and Cinzia Giuntoli Royal Edinburgh Hospital

${ }^{*}$ Corresponding author.

doi: 10.1192/bjo.2021.205

Aims. To assess junior doctors understanding of the law surrounding the use of The Mental Health (Care and Treatment) (Scotland) Act 2003 (MHA) with a focus on assessing confidence and knowledge of the use of the emergency detention certificate (EDC). A secondary aim was to use these findings to develop a variety of educational tools to subsequently improve junior doctors understanding in using the MHA.

Method. We created and distributed a comprehensive electronic survey to 152 Foundation Year Two Doctors working in NHS Lothian, Fife and Borders in December 2020. We subsequently interviewed 20 respondents to enquire about additional resources needed to improve knowledge of the MHA. Following on, we completed worked EDC exemplars, created an easily accessible guide with step-by-step instructions on implementing an EDC and devised a checklist pro-forma that can be accessed and inserted into a patient's electronic notes to ensure all necessary steps are completed for the EDC.

Result. 51 doctors (34\%) responded to our survey, of which 10 (19\%) had previously worked in psychiatry and 16 (31\%) had previously completed an EDC. 27 respondents (52\%) reported a lack of self-confidence and knowledge and 26 (51\%) reported a lack of understanding in the legal processes as barriers faced when putting an EDC in place. $23(45 \%)$ respondents were unaware that a Mental Health Officer (MHO) must be contacted to grant an EDC. Respondents who had experience of working in psychiatry reported greater awareness of the MHA. From the focused interviews held, colleagues requested for worked EDC examples, an easily accessible checklist with relevant contact details and an 
electronic pro-forma for patients notes to assist with completing the relevant legal steps when implementing an EDC.

Conclusion. Our study identified a lack of confidence in understanding the MHA and completing an EDC. Our educational materials will provide an invaluable source of information for junior doctors, in particular those with little experience of the MHA. Importantly, our resources will ensure the legal aspects of implementing an EDC are both complied with and documented appropriately.

Prevalence, associated factors and prevention of burnout in psychiatry trainees in Central and North West London NHS Foundation Trust

Grace $\mathrm{Xia}^{*}$ and Ahrane Jayakumar

Central and North West London NHS Trust

${ }^{\star}$ Corresponding author.

doi: 10.1192/bjo.2021.206

Aims. To assess burnout, resilience, professional quality of life and coping mechanisms in Central and North West London psychiatry trainees

Objectives. To determine Key factors associated with stress and burnout in workplace Effects of burnout on patient care and doctors Coping mechanisms used by trainees

Background. Burnout is a well established condition that has been recently reported to affect a third of doctors. Psychiatrists in particular represents a high risk group among doctors for experiencing burnout, alcohol and drug use, posing suicide risk and other forms of work related stress.

Method. The study comprised of a cross sectional questionnaire survey which included measure of stress (General Health Questionnaire), burnout (Maslach Burnout Inventory), and satisfaction with medicine as a career and personality (Big Five). During October to December 2019, core trainee and specialty trainee doctors in CNWL were asked to complete an online survey via emails.

Result. We collected data from 50 CNWL psychiatry trainees. The sample consisted of 20 females (40\%) and 30 males (60\%). Ages varied from $26-58$ years old, with a median age of 28 . Core trainees (CT1-3) were recorded as $72 \%$ and specialty trainees at $28 \%$.

Of those who responded, around half of the trainees (52\%) experienced high levels of stress outside of work in their personal life. The most common causes that trainees felt makes psychiatry a stressful profession were violence and fear of violence, limited resources, dealing with confrontational patients, inability to affect systemic change and increasing culture of blame. Around half of respondents (54\%) felt that they have experienced burnout but only $26 \%$ of respondents knew where to go to find resources to help cope with burnout. Physical exercise and speaking to colleagues were the most common coping mechanisms used by trainees to deal with stress.

Free text responses on what can be improved in workplace to enhance a positive experience of work included improving multidisciplinary interactions, easily accessible resources and increasing staffing levels. $74 \%$ of respondents felt they continued to care about what happens to patients regardless of working conditions. Conclusion. Half of CNWL trainee doctors who responded have experienced burnout. Some factors associated with stress and burnout in doctors are unique to psychiatry profession. Free text responses were useful in identifying areas for improvement in work places and useful coping mechanisms, which can be used to inform prevention and implement interventions to tackle burnout.
Suicidality in patients with bipolar depression: findings from a lower middle-income country

Siqi Xue ${ }^{1 *}$, John Hodsoll ${ }^{2}$, Ameer Bukhsh Khoso ${ }^{3}$, Muhammad Omair Husain ${ }^{4}$, Imran B Chaudhry, Allan H Young ${ }^{2}$, Juveria Zaheer ${ }^{4}$, Nusrat Husain ${ }^{5}$ and Benoit Mulsant ${ }^{1}$

${ }^{1}$ University of Toronto; ${ }^{2}$ king's college london; ${ }^{3}$ Pakistan Institution of Living and Learning; ${ }^{4}$ Centre for Addiction and Mental Health and ${ }^{5}$ University of Manchester

${ }^{\star}$ Corresponding author.

doi: 10.1192/bjo.2021.207

Aims. Among low- and middle-income countries (LMICs), bipolar disorder is recognized as one of the leading causes of disease burden for adults and is associated with marked suicide risk. There are limited data on suicidal ideation in bipolar disorder from LMICs. This study presents cross-sectional data on the prevalence of suicidality and associated patient characteristics among patients with bipolar depression in Pakistan, a lower-middle income country and the fifth most populous country in the world.

Method. Participants were recruited through outpatient psychiatric clinics in between 2016-2019 in Karachi, Lahore, Hyderabad and Rawalpindi between 2016-2019. Participants were aged 18 to 65 years with a known diagnosis of bipolar disorder and currently in a depressive episode. Suicidality was assessed using the suicide item of the 17-item Hamilton Depression Rating Scale (HAM-D) and levels of severity were categorized as absent, mild/moderate, or severe. Biometric data and biomarkers were obtained. Descriptive statistics were used to describe prevalence and proportional odds regression models were applied to establish correlates to suicidal ideation.

Result. Among the 266 participants, $67 \%$ indicated suicidality of any level and $16 \%$ endorsed severe suicidality. Lower body mass index $(\mathrm{BMI})(\mathrm{OR}=0.93,95 \% \mathrm{CI}=0.88-0.98)$, higher HAM-D score $(\mathrm{OR}=1.29,95 \% \mathrm{CI}=1.16-1.43)$, lower $\mathrm{C}$-reactive protein (CRP) level ( $\mathrm{OR}=0.53,95 \% \mathrm{CI}=0.40-0.70)$, and increased number of inpatient hospitalizations $(\mathrm{OR}=1.16,95 \% \mathrm{CI}=1.03-1.31)$ were identified as significant predictors of suicidality in the fully adjusted regression model. No patient demographic data, including age, gender, marital status, socioeconomic status, and years of education were associated with severity of suicidality.

Conclusion. There exists a high prevalence of suicidal ideation among patients with bipolar depression in Pakistan. Our findings add to the limited literature on suicidality in bipolar disorder in the LMIC context and suggest roles of biological variables such as BMI and CRP level in predicting suicidal ideation and potentially suicidal behaviours in bipolar depression. More studies are needed to see whether such findings can be replicated in other similar LMIC settings, and to explore potential physiological pathways linking BMI, inflammatory biomarkers and suicidality in bipolar disorder.

\section{Implementation of treatment escalation plans in a community psychiatric hospital}

Anne Yan Ting Chua ${ }^{1 \star}$, Adnaan Ghanchi ${ }^{1}$, Jessica Grayston ${ }^{1}$, Nida Yasmeen ${ }^{2}$, Sean Insigne ${ }^{2}$, Stephen Woolford ${ }^{1}$, Sahan Wijayaweera ${ }^{2}$, Harnish P Patel ${ }^{2}$, Jay Amin ${ }^{1}$ and Sangeeta Makh ${ }^{1}$

${ }^{1}$ University of Southampton and ${ }^{2}$ Southern Health NHS Foundation Trust

${ }^{\star}$ Corresponding author.

doi: 10.1192/bjo.2021.208 